\title{
TRYPANOSOMA RANGELI: SEM PROFILES DURING THE MIGRATION FROM THE MID-GUT TO THE SALIVARY GLANDS OF A REDUVIID BUG RHODNIUS PROLIXUS VIA DORSAL VESSEL
}

\author{
Maria Carlota MonRoY ${ }^{1}$ And Masataka KogA² \\ Received August 25, 1997/Accepted October 3, 1997
}

\begin{abstract}
Metamorphosis of Trypanosoma rangeli during the migration in the insect host, Rhodonius prolixius, was first documented by scanning electron microscopy (SEM). After infection, the parasites reach the insect alimentally tract and then they penetrate into the mid-gut and eventually emerge in the hemolymph of the abdominal cavity. From the abdominal cavity, both the parasites and the hemolymph are propelled to the thorax by the dorsal vessel (aorta pump), the end of which is terminated very near the salivary glands. Thus, once these parasites invade the abdominal cavity, they finally appear in the salivary glands via the transport by the dorsal vessel. The parasites gradually change themselves in shape during the migration. In the present study, we focused on the morphological characteristics of $T$. rangeli at individual stage in the vector by SEM and revealed some new findings in morphology and confirmed a migration route of this species. These results suggest that Guatemalan $T$. rangeli is mainly transmitted through the salivary gland.
\end{abstract}

Key words: Trypanosoma rangeli, insect vector, circulatory system, hemolymph, parasite ultrastructure, SEM

\section{INTRODUCTION}

The prevalence frequency of Trypanosoma rangeli Tejera, 1920 was from 6 to 10 times or more than of $T$. cruzi in certain regions of Central America (Sousa, 1972). This parasite was first detected in humans in Guatemala (De Leon, 1949) and later found in some reduviid vectors and wild reservoirs (Barrett and Oliveira, 1977). It is not easy to differentiate the species among the flagellar forms of trypanosomes in vectors (Vallejo et al., 1988). Several immunological trials for differtiating species have failed because of crossreactivity (Anthony et al., 1979; Lopez et al., 1981; O'Daly et al., 1994). However it is necessary to distinguish between the pathogenic and non-pathogenic trypanosomes for epidemiological investigations. On this context, some trials were performed by using various biological methods to distinguish $T$. cruzi from $T$. rangeli, as seen in the reports of Anthony et al. (1981), Marinkelle et al. (1985), Vallejo et al. (1993) and Mello et al. (1995). Especially, the last one has reported remakable defferences that $T$. rangeli in the homocoel of $R$. prolixus multiplied very rapidly, while $T$. cruzi showed no division and disappeared eventually from the homocoel of the vector.

Though several controversial data have been observed regarding to the transmission routes, namely, either salivary route or fecal one (D'Alessandro, 1963; Anez, 1982; Hoare, 1972) and whether T. rangeli persisted intracellularly as the amastigote form or not in the insect gut (Tobie, 1961; Anez, 1983; Hecker et al., 1990), nontheless it was commonly accepted that this proto-

1 Universidad de San Carlos de Guatemala, Facultad de Ciencias Quimicas y Farmacia, Escuela de Biologia, Ciudad Universitaria, Zona 12, Guatemala City, Guatemala, C.A.

2 Department of Parasitology, Faculty of Medicine, Kyushu University, Fukuoka 812-82, Japan Authors correspondence should be sent to M. Koga, Kyushu University, Fukuoka 812-82, Japan E-mail address: (Masataka Koga) 
zoan parasite was pathogenic to its vector insect (Schaub, 1994; Molyneuz, 1983).

The present paper reports the metamorphism of $T$. rangeli during the migration from the mid-gut through the abdominal cavity and dorsal vessel to the salivary glands of $R$. prolixus. The morphological alteration of the parasite in the mid-gut, hemolymph and salivary glands were traced by a scanning electron microscopy (SEM).

\section{MATERIALS AND METHODS}

Insect and parasite

A laboratory colony of $R$. prolixus which was originally obtained in Zacapa, Guatemala, C.A. in 1993 and maintained thereafter was used. Parasite was of Guatemalan $T$. rangeli strain recently isolated from the salivary gland of $R$. prolixus.

\section{Infection and Dissection}

Young nursing mice of the White Swiss strain were infected with $T$. rangeli by injecting the contents of the salivary gland of $R$. prolixus into the peritonium. When high parasitemia appeared about 7 days after infection
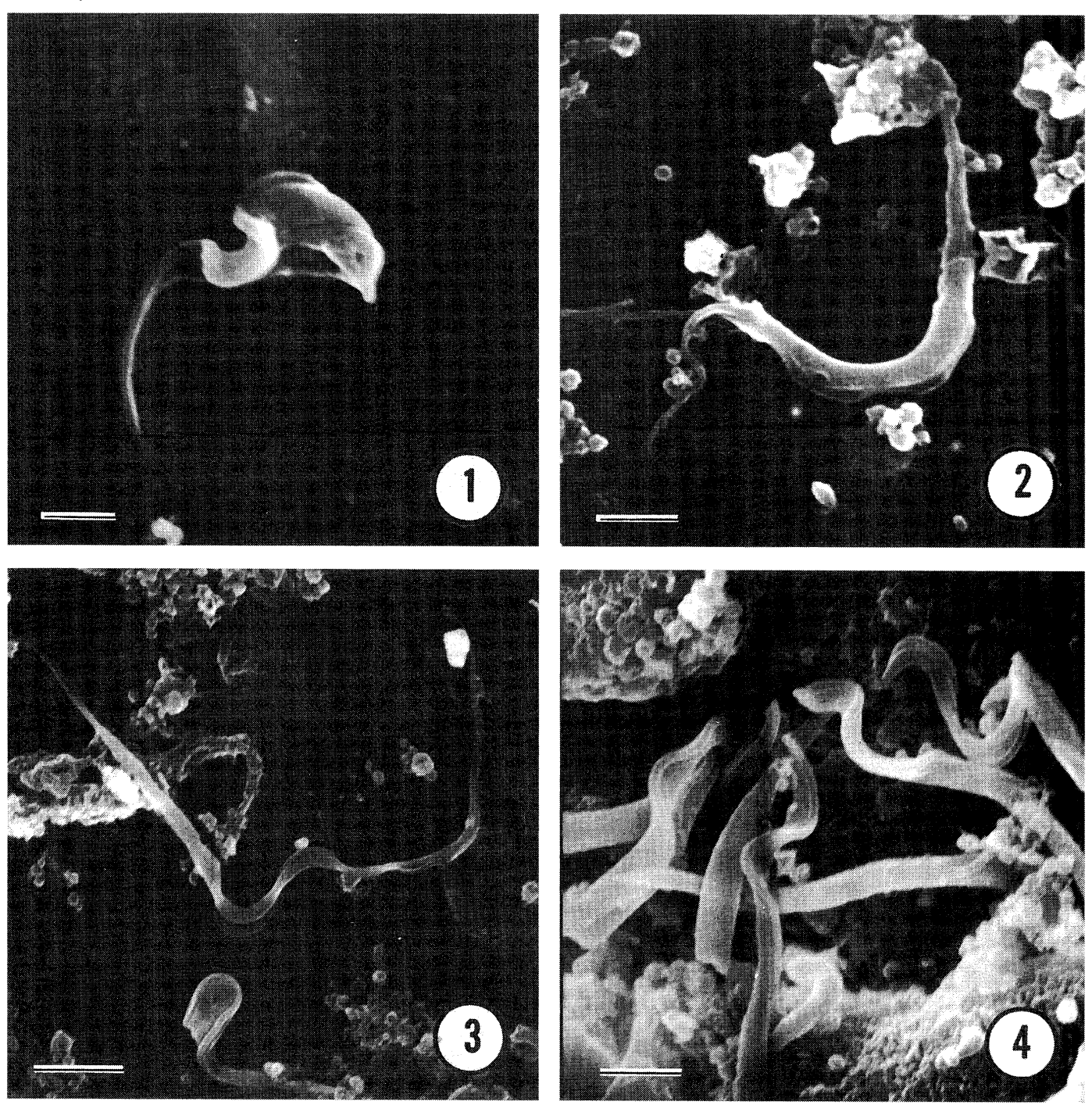

Figure 1 Trypanosoma rangeli metacyclic trypomastigote in the salivary gland. $\mathrm{Bar}=1 \mu \mathrm{m}$.

Figure 2 Trypomastigote of $T$. rangeli from the hemolymph. Bar $=2 \mu \mathrm{m}$

Figure 3 Long epimastigote of $T$. rangeli from the hemolymph. Bar $=3 \mu \mathrm{m}$

Figure 4 Massive penetration of epimastigotes of $T$. rangeli in the slender midgut. $\mathrm{Bar}=1 \mu \mathrm{m}$ 
in the mice, various stages of $R$. prolixus reared in laboratory conditions were fed on the blood of infected mice.

Periodically the infected triatomine bugs were dissected, and the hemolymph, salivary glands and gut contents were examined under a light microscopy. The heavily infected bugs were processed for SEM examination. Concurrently Giemsa's stained smear specimens of various tissues were prepared at $\mathrm{pH} 6.5$ for $2 \mathrm{hrs}$.

\section{Material preparation for SEM}

Dissected tissues were washed in saline solution and fixed over night in $3 \%$ glutaraldehyde in $0.1 \mathrm{M}$ sodium cacodylate buffer, washed $1 \mathrm{hr}$ in phosphate buffer and postfixed in osmium tetroxide in 2 hours. They were then dehydrated in a graded series of ethanol, dried at critical point in a Hitachi HCP dryer, coated with gold in a JEOL ion-spatter coater, and examined under JEOL U-3 scanning electron microscope at $15 \mathrm{kv}$.

In addition, the hemolymph and the excrement were placed on a round cover glass previously coated by polyL-Lysine solution and then dried. The glass was gently placed and attached on the top of a stub. The materials
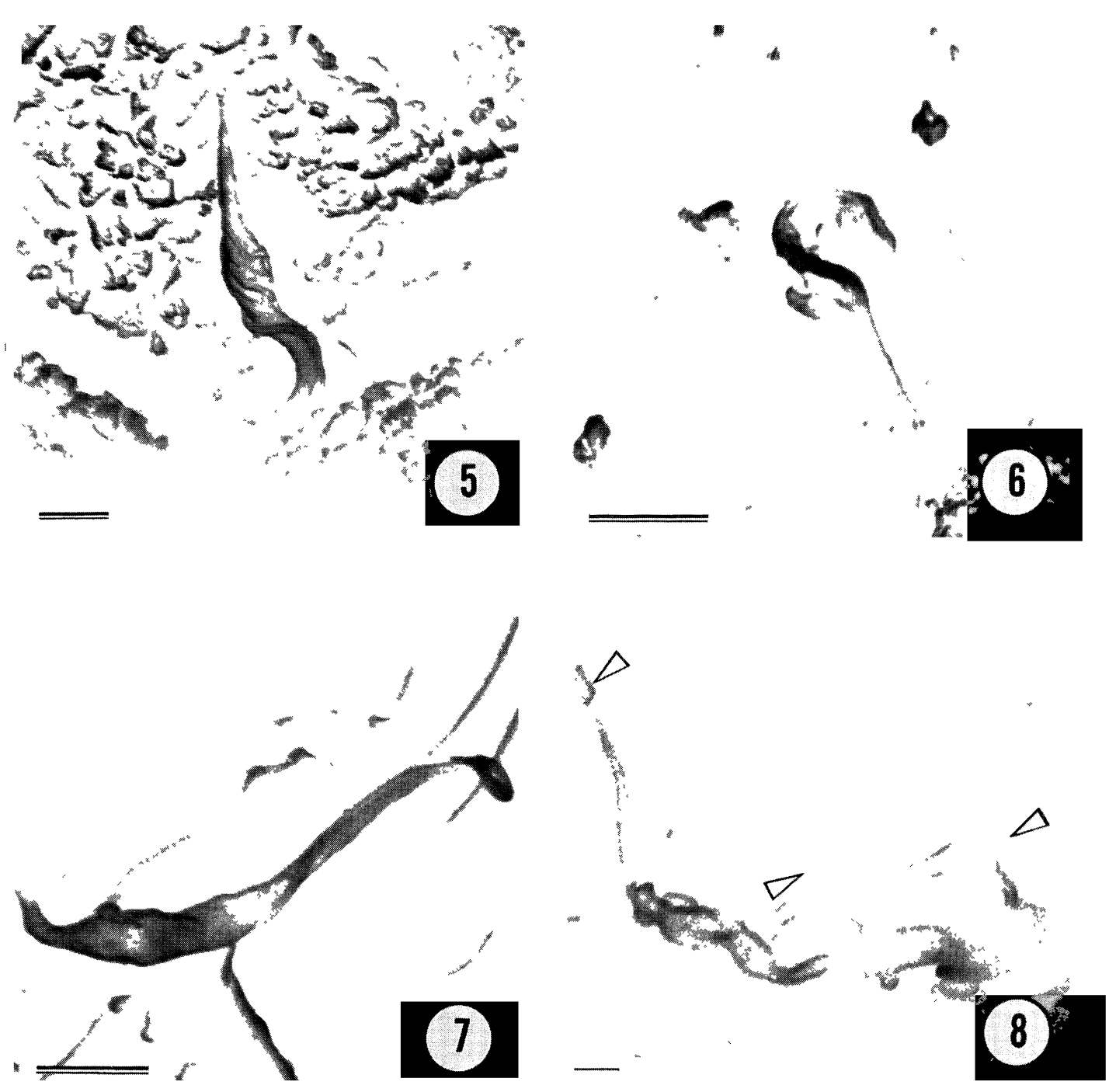

Figure 5 Final phase of penetration to the mid-gut of the host. Posterior portion of epimastigote of T. rangeli is evident. $\mathrm{Bar}=2 \mu \mathrm{m}$

Figure 6 Spheromastigote of $T$. rangeli in the vector's gut. Bar $=2 \mu \mathrm{m}$

Figure 7 Epimastigote of $T$. rangeli in the dorsal vessel of $R$. prolixus; the contractile elements and striated muscle fibers were seen $\operatorname{Bar}=2 \mu \mathrm{m}$

Figure 8 Epimastigote of $T$. rangeli lying in the dorsal vessel attached with some bacteria (arrowheads) which are commonly found in the gut of the vector. Bar $=1 \mu \mathrm{m}$ 
were allowed to stay on the glass for $1 \mathrm{hr}$ and were then fixed with glutaraldehyde. Post-fixation by osmium tetraoxide was omitted before SEM examination, because these small parasites often disappeared from the tissues of the insect by the conventional SEM specimen preparing procedure.

\section{RESULTS}

The flagella in all stages of $T$. rangeli were clearly seen in insects. There were three shapes in trypomastigotes in the insect, one in the feces, another in the hemolymph and the last one in the salivary gland. Fig. 1 showed the metacyclic trypomastigote from the salivary glands. The parasite from this gland is the smallest in all and somewhat stout. On the contrary, the trypomastigotes in the abdominal cavity were bigger and slender (Fig. 2), and very similar to those in the feces. The trypomastigotes in the feces had't infectious ability to mice in experimental infection.

Epimastigotes were dominant in the insect hosts. Their bodies were also short, medium and very long in size. However the trypomastigotes distinguished from the epimastigotes by their shape and size. They often segregated in a same host.

Fig. 3 showed one of the longest epimastigotes in the mid-gut.

Fig. 4 showed that the medium sized epimastigotes which were penetrating into the gut epithelium. Fig. 5 showed the last step of the penetration of epimastigote to the mid-gut. As seen in this figure, the body penetration elicited intense spiral movement which was accelerated with its flagellum. This flagellum movement seemed to play a very important role in the penetration. The trigger of the invasion is unknown. Fig. 6 shows the spheromastigote that is developing from the amastigote (which means a round form parasite in the mig-gut epithelium or hemocyte described by Hecker et al., 1990) and is just shifting to epimastigote. This spheromastigote is also a very small in size.

Fig. 7 also showed the epimastigote on the contractile fibers of the folded muscle tissues in the dorsal vessel. Once the epimastigotes intrude into the host's abdominal cavity filled with hemolymph, multiplication occurs there and eventually a great number of parasites is able to be found in the cavity. The dorsal vessel of the bugs absorbs the hemolymph into the aorta which acts as the pumping organ. By this means, the vessel soaked up the parasites together with the liquid and conveyed to the thorax region. As the vessel is terminated very near to the salivary glands in the thorax, the parasites conse- quently seemed to have penetrated into the salivary glands by themselves. Fig. 8 shows the parasite surrounded by some bacteria (arrowheads) that were probably brought with the parasite from the intestine of the insect into the vessel.

\section{Discussion}

Some authors reported that $T$. rangeli trypomastigote from feces could infect mice (D'Alessandro, 1963; Hoare, 1972), while the other noted that only the parasites from the salivary glands had infectivity (Tobie, 1961; Anez, 1982). Present study showed that there were some differences in shapes and sizes of the trypomastigotes and epimastigotes in various regions of the vector insect, and this rapid morphological changes are related to this parasite infectivity. In mice infection with trypomastigotes from the salivary glands, we always saw parasitemia, whereas we could not see it with the parasites from the bug excrements. On the other hand, mice infected with the parasites from the hemolymph, no parasitemia occurred. These results agreed with the findings of Tobie (1961) and Anez (1982). From these findings, we want to suggest that the salivary glands play an important role for the transmission of $T$. rangeli to mice. It is reported that there is a difference in the tissue affinity in bugs between $T$. rangeli and $T$. cruzi; $T$. rangeli favors to live in the slender mid-gut, whereas $T$. cruzi prefers the rectal sac in the same host. Furthermore $T$. cruzi attached to the rectal wall tightly (Zeledon et al., 1984), while $T$. rangeli crawled freely in the mid-gut. In addition, the epimastigotes of $T$. rangeli in the hemolymph and the dorsal vessel were provided with very long and slender body, which is one of the characteristics of this species.

For these characteristcs mentioned above, we facilitated the differentiation of each species by careful separation of the vector's hemolymph, salivary glands, rectal sac and the slender mid-gut.

Spheromastigotes have been reported in both species of $T$. cruzi and $T$. rangeli (De Lucena et al., 1973; Vallejo et al., 1988; Marinkelle et al., 1985) as a shifting stage from amastigote to epimastigote.

Present study is the first report for documenting spheromastigote form of $T$. rangeli by SEM.

It has been described that in $T$. rangeli infection, the symbionts (some bacteria) were small in number in the hemolymph of the bugs (Schaub, 1994). In the present study, however, we observed many bacteria in the hemolymph and even in the circulatory system of the vector by SEM. This is probably because of the tissue 
damage of the insect gut due to massive invasion of the parasites.

In this study, trypomastigotes in the salivary glands as well as some stages of epimastigotes and spheromastigotes of $T$. rangeli in $R$. prolixus, the vector were first demonstrated by SEM. The authors here emphasize that flagella of trypanosomes seem to play a major role in the penetration to the gut wall.

\section{ACKNOWLEDGEMENT}

We are grateful to Prof. I. Tada, Department of Parasitology, Faculty of Medicine, Kyushu University, Japan for his critical reading the manuscripts. This work was supported by JICA-Guatemala Tropical Disease Project (GJET-125).

\section{REFERENCES}

1) Anez, N. (1982): Studies on Trypanosoma rangeli Tejera 1920 IV. A reconsideration of its systematic position. Mem. Inst. Oswaldo Cruz, 77, 405-515

2 ) Anez, N. (1983): Studies on Trypanosoma rangeli Tejera 1920. V. Developmental pattern in the alimentary canal of Rhodnius prolixus. Mem. Inst. Oswaldo Cruz, 78, 183191

3 ) Anthony, R.L., Johnson, C.M. and Sousa, O.E. (1979): Use of Micro-ELISA for quantitativating antibody to Trypanosoma cruzi and Trypanosoma rangeli. Am. J. Trop. Med. Hyg., 28 (6), 969-973

$4)$ Anthony, R.L., Cody, T.S. and Constantine, N.T. (1981): Antigenic differentiation of Trypanosoma cruzi and Trypanosoma rangeli by means of monoclonalhybridoma antibodies. Am. J. Trop. Med. Hyg., 30 (6), 1192-1197

5 ) Barrett, T.V. and Silva de Oliveira, T. (1977): A trypanosome, indistinguishable from Trypanosoma rangeli, in the hemolymph of Rhodnius domesticus from Brazil. Trans. Roy. Soc. Trop. Med. Hyg., 71(5), 445446

6 ) D'Alessandro, A. (1963): The life cycle of Trypanosoma rangeli. In triatomid bugs as it occurs in nature. Bull. Tulane Med. Fac., 23, 21-31

7 ) De Leon, R.J. (1949): El Trypanosoma rangeli observado en sangres humanos en Guatemala. Publicaciones del Instituto de Investigaciones Cientifica, Guatemala, 3, 134

8 ) De Lucena, D.T. and Bergetti, J.G. (1973): Infeccion natural de Panstrongylus megistus (Burmeister, 1835) por Trypanosoma rangeli (Tejera, 1920), no interior de estado de Alagoas. Rev. Inst. Med. Trop. San Paulo., 15 (4), 171-178

9 ) Hecker, H., Schwarzinbach, M. and Rudlin, W. (1990): Development and interactions of Trypanosoma rangeli in the reduviid bug Rhodnius prolixus. Parasitol. Res., 76, 311-318

10) Hoare, C. (1972): The trypanosomes of mammals. Blackwell Sci. Pub. Oxford, pp. 288-314

11) Hudson. L., Guhl, F., De Sanches, N., Bridge, D., Jaramillo, C. A. and Young, A. (1988): Longitudinal studies of the immune response of Colombian patients infected with Trypanosoma cruzi and $T$. rangeli. Parasitology, 96, 449-460

12) Lopez, D.J., Caulada, Z., Clara, L. Barbeiri and Plessman Camargo, E. (1981): Cross-reactivity between Trypanosoma cruzi and insect trypanosomatids as a basis for the diagnosis of Chagas disease. Am. J. Trop. Med. Hyg., 30 (6), 1183-1188

13) Mello, C.B., Garcia, E.S., Ratcliffe, N.A. and Azambuja, P. (1995): Trypanosoma cruzi and Trypanosoma rangeli: Interplay with hemolymph components of Rhodnius prolixus. J. Inverteb. Pathol., 65, 261-268

14) Maminkelle, C.J., Vallejo, G.A., Guhl, F. and De Sanchez, N. (1985): Diferenciacion entre Trypanosoma cruzi y Trypanosoma rangeli en el intestino del vector Rhodnius prolixus, en base al comportamiento de estos flagelados frente a la activedad litica del complemento. Rev. Lat-Amer. Microbiol., 27, 21-25

15) Molyneus, D.H. (1983): Host-parasite relationship of Trypanosomatidae in vectors. Vol. 1, pp. 117-148. K.F. Harris, (ed.). Current Topics In Vector Research. Praeger Publisher, New York

16) O'Daly, J.A., Carrasco, H., Fernandez, V. and Ro druiguez, M.B. (1994): Comparison of chagasic and nonchagasic myocardiopathies by ELISA and immunoblotting with antigens of Trypanosoma cruzi and Trypanosoma rangeli. Acta Trop., 56, 265-287

17) Schaub, G.A. (1994): Pathogenicity of Trypanosomatids on insects. Parasitology Today, 10, 463-468

18) Sousa, O.E. (1972): Anotaciones la enfermedad de Chagas en Panama. Frecuencia y distribucion de Trypanosoma cruzi y Trypanosoma rangeli. Rev. Biol. trop., $20(2), 167-179$

19) Tobie, E.J. (1961): Experimental transmission and biological comparison of strains of Trypanosoma rangeli. Exp. Parasitol., 11, 1-9

20) Vallejo, G., Marinkelle, C., Guhl, F. and De Sanches, N. (1988): Comportamiento de la infeccion y diferenciacion morfologica entre Trypanosoma cruzi y $T$. rangeli en el intestino del vector Rhodnius prolixus. Rev. Brazil Biol., $48,577-587$

21) Vallejo, G., Chiari, E., Macedo, A. and Pena, S.D. (1993): A simple laboratory method for distinguishing between Trypanosoma cruzi and Trypanosoma rangeli. Trans. Roy. Soc. Trop. Med. Hyg., 87, 165-166

22) Zeledon, R., Bolanos, R. and Rojas, M. (1984): Scanning electron microscopy on the final phase of the life cycle of Trypanosoma cruzi in the insect vector. Acta Trop., 41, 39-43 